\title{
The effect of virtual reality on visual vertigo symptoms in patients with peripheral vestibular dysfunction: A pilot study
}

\author{
M. Pavlou ${ }^{\mathrm{a}, *}$, R.G. Kanegaonkar ${ }^{\mathrm{b}}$, D. Swapp ${ }^{\mathrm{d}}$, D.E. Bamiou ${ }^{\mathrm{b}, \mathrm{c}}$, M. Slater ${ }^{\mathrm{d}, \mathrm{e}}$ and L.M. Luxon ${ }^{\mathrm{b}}$ \\ ${ }^{\mathrm{a}}$ Centre of Human and Aerospace Physiological Sciences, King's College London, London, UK \\ ${ }^{\mathrm{b}}$ Department of Neuro-Otology, National Hospital for Neurology and Neurosurgery, London, UK \\ ${ }^{\mathrm{c}}$ Ear Institute, University College London, London, UK \\ ${ }^{\mathrm{d}}$ Department of Computer Science, University College London, London, UK \\ ${ }^{\mathrm{e}}$ Faculty of Psychology, ICREA-University of Barcelona, Barcelona, Spain
}

Received 15 April 2010

Accepted 23 October 2012

\begin{abstract}
Individuals with vestibular dysfunction may experience visual vertigo (VV), in which symptoms are provoked or exacerbated by excessive or disorientating visual stimuli (e.g. supermarkets). VV can significantly improve when customized vestibular rehabilitation exercises are combined with exposure to optokinetic stimuli. Virtual reality (VR), which immerses patients in realistic, visually challenging environments, has also been suggested as an adjunct to VR to improve VV symptoms. This pilot study compared the responses of sixteen patients with unilateral peripheral vestibular disorder randomly allocated to a VR regime incorporating exposure to a static (Group S) or dynamic (Group D) VR environment. Participants practiced vestibular exercises, twice weekly for four weeks, inside a static (Group S) or dynamic (Group D) virtual crowded square environment, presented in an immersive projection theatre (IPT), and received a vestibular exercise program to practice on days not attending clinic. A third Group D1 completed both the static and dynamic VR training. Treatment response was assessed with the Dynamic Gait Index and questionnaires concerning symptom triggers and psychological state. At final assessment, significant betweengroup differences were noted between Groups $\mathrm{D}(p=0.001)$ and $\mathrm{D} 1(p=0.03)$ compared to Group S for VV symptoms with the former two showing a significant $59.2 \%$ and $25.8 \%$ improvement respectively compared to $1.6 \%$ for the latter. Depression scores improved only for Group S $(p=0.01)$ while a trend towards significance was noted for Group D regarding anxiety scores $(p=$ 0.07). Conclusion: Exposure to dynamic VR environments should be considered as a useful adjunct to vestibular rehabilitation programs for patients with peripheral vestibular disorders and VV symptoms.
\end{abstract}

Keywords: Vestibular rehabilitation, virtual reality, visual vertigo

\section{Introduction}

Vestibular rehabilitation incorporating habituation, adaptation, balance and gait exercises is currently considered the standard of care for patients with peripher-

* Corresponding author: Dr. Marousa Pavlou, King's College London, Centre of Human and Aerospace, Physiological Sciences, Room 3.5 Shepherd's House, London, SE1 1UL, UK. Tel.: +44 207848 6328; Fax: +44 207848 3275; E-mail: marousa.pavlou@kcl.ac.uk. al vestibular disorders regardless of age and symptom duration $[8,17,44]$. Numerous studies show significant improvements in subjective symptoms, dynamic visual acuity, gait and postural stability $[5,8,16,26]$. However visual vertigo (VV) [7] symptoms or visually induced dizziness [4] only improve when vestibular rehabilitation is combined with additional exposure to optokinetic stimulation [31].

Patients with VV report an exacerbation or provocation of postural instability and vestibular symptoms 
in visually rich environments, such as crowds, moving traffic or escalators [13] which cannot be explained by a susceptibility to motion sickness or anxiety [20,30]. It has been suggested that VV results from an over reliance on visual cues for perception and postural control (i.e. visual dependence) $[7,13]$ with findings showing a stronger effect of disorienting visual stimuli (tilted or rotating visual surroundings) on verticality perception and postural stability in patients with peripheral vestibular dysfunction plus VV, compared to those without VV [7,30].

It is believed that graded exposure to optokinetic stimulation results in a decreased over-reliance on visual input for perceptual and postural responses [13,32]. Recent findings show that short-term repeated exposure to visuo-vestibular exercises induces adaptive changes, decreasing (improving) the magnitude of visual dependency in healthy controls [32]. Furthermore, when simulator-based optokinetic stimulation exposure via whole-body or visual environment rotators is combined with a customized vestibular exercise regime, significantly greater improvements are noted for VV symptoms and postural instability post-treatment compared to customized exercises alone [31].

Virtual reality may be a useful adjunct to vestibular rehabilitation, as it may help generate realistic and interactive visual environments in which the patient is immersed and which may facilitate adaptation and desensitization [35]. A feasibility study investigated the use of spatially immersive virtual reality exposure in healthy adults and patients with a peripheral vestibular deficit and found postural sway to be increased during virtual reality exposure in participants with and without a vestibular deficit [35]. Furthermore a preliminary study involving placing patients with chronic vertigo due to a vestibular disorder, in an immersive, computer generated moving visual scene that interacted with their head movements, showed improvements in vestibulo-ocular reflex gain and dizziness handicap after 5 days [39]. However, no studies to date have assessed the impact of virtual reality on VV symptoms.

The aim of the current study was to assess the potential benefits of combining vestibular exercises with exposure to an immersive visually challenging virtual environment on VV symptoms in patients with chronic symptoms secondary to vestibular pathology.

\section{Materials and methods}

\subsection{Participants}

The research project received approval from the $\mathrm{Na}-$ tional Hospital for Neurology and Neurosurgery and the Institute of Neurology Joint Research Ethics Committee. Patients were recruited from the Department of Neuro-otology at the National Hospital for Neurology and Neurosurgery via review of clinic letters between December 2002 and January 2004. All had a clinical neuro-otological and oculomotor assessment, bithermal caloric test, electronystagmography including sinusoidal and step rotational responses and pure tone audiogram, as per the standard departmental clinical protocol.

Inclusion criteria were a) age between 18-75 years of age, b) a confirmed peripheral vestibular deficit (on the basis of the caloric test and/or rotational tests on the ENG) and c) a history of an acute onset of vertigo, unaccompanied by any auditory, systemic and neurological symptoms or signs, consistent with a diagnosis of vestibular neuritis.

Patients with a diagnosis of migrainous vertigo, Menière's disease, benign paroxysmal positional vertigo, central vestibular disorders, other neurological disorders, significant systemic illness or psychiatric disorders were excluded.

Seventeen patients agreed to participate in the study, but one did not complete the trial due to non- compliance (Group S) and is not included in the analysis. Patients were initially randomly allocated into one of two treatment groups (Group D = Dynamic virtual reality; Group $S=$ Static virtual reality image). Eleven patients were allocated into Group S and 5 into Group D. The first five participants from Group $S$ then also received rehabilitation with dynamic virtual reality (Group D1) after a period of at least two months to avoid any potential "carry-over" effects from the initial treatment. No significant between-group differences were noted for age or baseline data between Groups $\mathrm{S}$ and D except for symptom duration ( $\mathrm{U}=9.5, z=-2.04, p=0.04$ ) with Group D showing a significantly longer average duration (Table 1).

\subsection{Intervention program}

\subsubsection{Virtual reality}

All patients attended twice weekly 45 minute therapy sessions for four weeks.

\section{i. Description of virtual reality equipment and environment \\ Virtual reality exposure took place within the ReaCTor $^{\mathrm{TM}}$ in the Department of Computer Science, University College London. The ReaCTor is an im- mersive projection theatre (IPT) of a type commonly}


Table 1

Participant characteristics at baseline

\begin{tabular}{lccc}
\hline & Group S $(n=11)$ & Group D $(n=5)$ & Group D1 $(n=5)$ \\
\hline Age (y) (mean, range) & $42.1(28-54)$ & $42.0(25-51)$ & $39.8(29-51)$ \\
$\begin{array}{l}\text { Gender (n) } \\
\text { Female, n (\%) }\end{array}$ & $4(36 \%)$ & $3(60 \%)$ & $2(40 \%)$ \\
$\quad$ Male, n (\%) & $7(64 \%)$ & $2(40 \%)$ & $3(60 \%)$ \\
$\begin{array}{l}\text { Symptom duration (mo) } \\
\text { (mean, range) }\end{array}$ & $40.7(6-88)$ & $86(50-156)$ & $37.6(12-78)$ \\
\hline
\end{tabular}

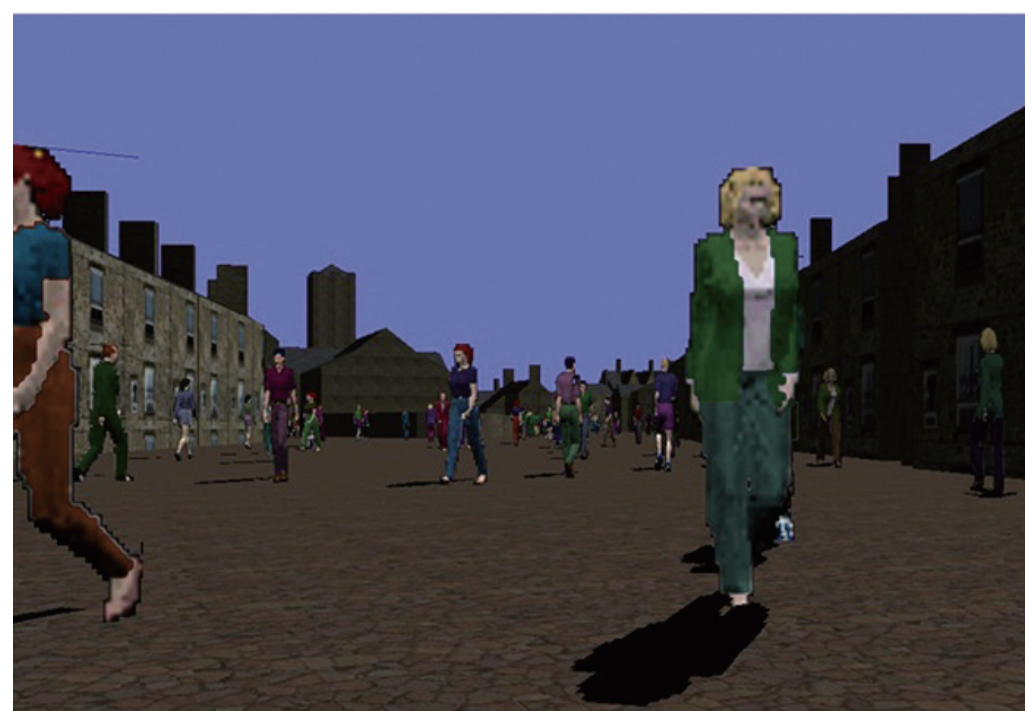

Fig. 1. The virtual crowded street scene used within the University College London ReAcTor ${ }^{\mathrm{TM}}$. (Colours are visible in the online version of the article; http://dx.doi.org/10.3233/VES-120462)

referred to as a CAVE [9], consisting of 3 rear-projected vertical screens (each $3 \mathrm{~m} \times 2.2 \mathrm{~m}$ ) arranged seamlessly around a front-projected horizontal floor screen $(3 \mathrm{~m}$ $\times 3 \mathrm{~m}$ ). Users were presented with high-resolution 3D stereo images, seamlessly integrated across all four screens to produce one continuous image. 3D stereo is achieved by the users wearing Shutterglasses (Crystaleyes $\left.{ }^{\circledR}\right)$ throughout their exposure within the IPT. The shutterglass lenses alternately block the left and right images of the stereo pair presented to the user by the projection system at 90 frames per second, thus each eye receives image updates at $45 \mathrm{~Hz}$. The images were presented with reference to the user's viewpoint only, which was continuously updated via an Intersense IS-900 head tracking unit. As the subject moved, the position and orientation of the user's head (and thus eyes) was updated and the projected images altered accordingly.

The scene rendered in the IPT consisted of a crowded street that formed part of an artificial town (Fig. 1). Groups D and D1 were exposed to computer generated human forms walking within the street (each at randomly defined rates of $1 / 4$ to 4 feet per second for the entire period of the virtual reality exposure), whilst Group S was exposed to an identical, but static, three dimensional crowd within the same street. Continuous head tracking showed correct image perspective from the point of view of the subject in all conditions.

\section{ii. Exercises practiced during virtual reality exposure}

Each subject was asked to perform nine exercises in sequence as listed in Table 2. Each exercise lasted 2 minutes, but the patients were instructed to say 'stop' at any point if they felt they could not continue due to nausea or dizziness. Symptoms were graded at one minute (see below under outcome measures) and if none were induced, the participant was asked to increase the head and/or walking speed for each exercise accordingly.

\subsubsection{Home vestibular exercise program}

All participants were asked to continue with their previously prescribed Cawthorne-Cooksey exercise program and general conditioning program (i.e. a 1530 minute walk three times weekly). 
Table 2

Exercises performed during virtual reality exposure

Exercise 1 . Standing upright focusing on a stationary point at eye level in the distance

Exercise 2. Move head up and down while focusing on a stationary point in the distance

Exercise 3. Turn head left and right while focusing on a stationary point in the distance

Exercise 4. Looking left and right over one's shoulder as if one was looking for someone standing behind them

Exercise 5. Standing upright, on a foam cushion, focusing on a stationary point at eye level in the distance

Exercise 6. Focusing on a stationary point in the distance whilst marching on the spot

Exercise 7. Focusing on a point in the distance, taking three steps forward and back

Exercise 8. Focusing on a point in the distance, taking three steps forward and back whilst moving one's head from side to side

Exercise 9. Focusing on a point in the distance, taking three steps forward and back whilst moving one's head up and down

\subsection{Outcome measures}

Subjective VV, psychological symptoms and functional gait were assessed at baseline, prior to patient and therapist knowledge of treatment group, and at 4 weeks (end of treatment).

\subsubsection{Questionnaire measures}

i. The Situational Vertigo Questionnaire (SCQ) [13, 33] yields a normalized score between 0 (never) to 4 (always) and assesses the frequency of symptoms of dizziness, giddiness, light-headedness or unsteadiness induced by specific visually disorientating environments such as walking down a supermarket aisle. Scores $\geqslant 0.7 / 4$ indicate VV symptoms [30].

ii. The Beck Depression Inventory [2] is a 21 item self-report measure of the level of depressive symptoms experienced by a subject during the previous two weeks. Composite scores range between 0 and 63. Scores 9 and below are normal; 10-18 indicate a mild to moderate level of depression; 19-29 moderate to severe, and 36-63 indicate severe depression.

iii. The Beck Anxiety inventory [23] is a 21 item self report measure of the level of anxiety a person has experienced during the previous week. Composite scores range between 0-63. Scores 7 and below are indicative of minimal anxiety levels, 8-15 mild, 16-25 moderate, and scores above 26 indicate severe anxiety levels.

iv. The Fear Questionnaire [25] is a self assessment tool that provides a measure of the severity of fear experienced by participants to 23 specific scenarios. Four scores are obtained: A level of avoidance caused by a specific target phobia identified in writing by that individual (question 1 , score range $0-8$ ), a total phobia score indicating the extent of avoidance for 15 common phobias (e.g. agoraphobia, blood/ injury, social; questions 2-16, score range 0-120), a rating of associated anxiety and depression (questions 1822 , score range $0-40$ ) and a global phobia rating reflecting distress and avoidance (score range $0-$ 8). For the current study, only the total phobia score was tabulated to limit the number of statistical comparisons.

\subsubsection{Dynamic gait index}

This tool was developed to identify balance dysfunction and falls risk during eight dynamic gait tasks [1, 42]. Each task is rated between 0 (inability to perform task) to 3 (normal ability). The maximum score is 24 and scores below 19 are indicative of a high falls risk. All participants were reassessed prior to their final virtual reality rehabilitation session (to avoid the effect of any immediate gait change resulting from the virtual reality exposure) by an independent observer.

\subsubsection{Virtual reality exercise symptom scores}

After each exercise practised during virtual reality exposure, participants were asked to rate their symptoms based on a scale between 0 (no symptoms) to 3 (severe symptoms). A score of 3 was recorded, if patients were unable to complete the full two minutes. A cumulative score was calculated by adding the individual scores for each exercise performed during session 1 and 8 (final session) and was used as a further outcome measure for statistical analysis.

\subsection{Statistical analysis}

SPSS 17 (SPSS Inc., Chicago, USA) was used for statistical analysis. Data are presented as mean \pm SD. Between-group differences (Group S vs. Group D; Group D vs. Group D1; and the six participants who only completed Group S vs. Group D1) were determined using Mann-Whitney $U$ test. Within-group differences pre (baseline) and post (week 4) intervention were analyzed using Wilcoxon signed rank tests. Spearman's correlation assessed the relationship between age and symptom duration with baseline scores and pre-post treatment changes for objective and self-report measures. 
Table 3

Mean (SD) of outcome measures for Groups S (static virtual reality training) and D (dynamic virtual reality training)

\begin{tabular}{|c|c|c|c|c|}
\hline & \multicolumn{2}{|c|}{ Group $\mathrm{S}(n=11)$} & \multicolumn{2}{|c|}{ Group D $(n=5)$} \\
\hline & Pre & Post & Pre & Post \\
\hline SCQ & $1.28(0.75)$ & $1.26(0.90)$ & $1.54(0.50)$ & $0.63(0.25)^{* \dagger}$ \\
\hline Beck A & 13.55 (7.39) & $11.18(9.84)$ & $13.40(6.11)$ & $7.00(2.83)$ \\
\hline Beck D & $10.18(5.15)$ & $6.55(4.82)^{*}$ & $9.60(6.99)$ & $6.00(7.87)$ \\
\hline Total Phobia & 24.18 (13.95) & $21.64(12.78)$ & $23.80(19.46)$ & $17.80(12.78)$ \\
\hline DGI & $19.91(3.65)$ & $20.18(4.21)$ & $20.20(4.97)$ & $23.00(1.73)$ \\
\hline VRCESS & $15.00(4.58)$ & $14.82(4.96)$ & $12.20(1.10)$ & $5.00(3.10)^{* \dagger}$ \\
\hline
\end{tabular}

$\overline{\mathrm{SCQ}}=$ Situational Characteristic Questionnaire; Beck A = Beck Anxiety Scale; Beck D = Beck Depression Scale; DGI = Dynamic Gait Index; VRCESS = virtual reality cumulative exercise symptom score. ${ }^{*} p<0.05$ indicates significant improvement compared to the pre-intervention assessment; ${ }^{\dagger} p<0.05$ indicates a significant between-group difference.

Table 4

Mean (SD) of outcome measures for Groups D1 (both static and dynamic virtual reality training) and Group S participants who only received static virtual reality training

\begin{tabular}{|c|c|c|c|c|c|c|}
\hline & \multicolumn{4}{|c|}{ Group D1 $(n=5)$} & \multirow{2}{*}{\multicolumn{2}{|c|}{$\frac{\text { Group } \mathrm{S}(n=6)}{\text { Static virtual reality only }}$}} \\
\hline & \multicolumn{2}{|c|}{ Static virtual reality } & \multicolumn{2}{|c|}{ Dynamic virtual reality } & & \\
\hline & Pre & Post & Pre & Post & Pre & Post \\
\hline SCQ & $1.67(0.86)$ & $1.61(1.08)$ & $1.53(0.97)$ & $1.14(0.92)^{* \dagger}$ & $0.96(0.51)$ & $0.98(0.70)$ \\
\hline Beck A & $13.40(9.56)$ & $15.4(13.76)$ & $13.40(7.70)$ & $7.60(4.62)$ & $13.70(6.02)$ & $7.70(3.10)$ \\
\hline Beck D & $12.40(5.13)$ & $5.60(5.37)$ & $9.40(6.58)$ & $8.00(7.18)$ & $8.33(4.80)$ & $7.33(4.68)$ \\
\hline Total Phobia & $26.80(10.85)$ & $19.40(10.31)$ & $21.60(13.47)$ & $16.40(12.94)^{*}$ & $22.00(16.80)$ & $23.50(15.24)$ \\
\hline DGI & $22.00(1.87)$ & $21.60(3.29)$ & $22.20(2.05)$ & $23.80(0.45)$ & $18.17(3.97)$ & $19.00(4.82)$ \\
\hline VRESS & $11.60(4.04))$ & $12.60(3.36)$ & $14.00(3.94)$ & $5.25(5.44)^{\dagger}$ & $17.83(2.79)$ & $16.67(5.57)$ \\
\hline
\end{tabular}

${ }^{*} p<0.05$ indicates significant improvement compared to the pre-intervention assessment; ${ }^{\dagger} p<0.05$ indicates a significant difference betweengroups D1 following dynamic virtual reality rehabilitation and Groups S participants who only completed the static training.

\section{Results}

No significant correlation was noted between age and symptom duration with either baseline scores or pre-post treatment change for self-report measures and the DGI for any of the groups.

No significant differences were noted for Group D1 between pre- and post-treatment scores when exposed to a static virtual reality scene; furthermore no significant differences were noted between post-treatment scores with static virtual reality scene treatment and baseline scores pre-treatment with dynamic virtual reality after the "waiting" period (Table 4). At baseline prior to starting any virtual reality treatment, no significant differences were noted between Group S participants who completed both static and dynamic virtual reality training and those who completed only the former (Table 4).

\section{1. $S C Q$}

A significant between-group difference in SCQ symptom score change $(U=1.0, z=-3.00, p=$ 0.001 ) was noted with a $59.2 \%$ improvement for Group $\mathrm{D}$ compared to a $1.6 \%$ change for Group S (Fig. 1,
Table 3). The difference between Group D1 and the six participants who only completed Group $\mathrm{S}$ was also significant with a $25.8 \%$ improvement for the former ( $U=3.5, z=-2.10, p=0.03$; Table 4). A significant within-group difference was noted only for Groups D and D1 $(Z=-2.02, p=0.04$, Tables 3, 4). The difference between Groups D and D1 approached significance $(U=3.0, z=-1.98, p=0.06)$ with Group D showing the greater improvement (Table 3). For Group $\mathrm{D}$ all participants showed improvements compared to 4/5 for Group D1 and 7/11 for Group S.

\subsection{Becks anxiety and depression scale}

No significant between-group differences were noted for either the Beck's Anxiety or Depression scale. Within-group analysis showed a significant improvement for depression scores in Group $\mathrm{S}(Z=-2.68$, $p=0.01)$. A trend towards significance was noted for anxiety scores $(Z=-1.84, p=0.07)$ for Group D. For both the Becks Depression and Anxiety scales 4/5 patients in Group D and 9/11 in Group S showed improved scores while in Group D1 2/5 and 4/5 patients improved respectively. Descriptive data and statistics are shown in Tables 3 and 4. 


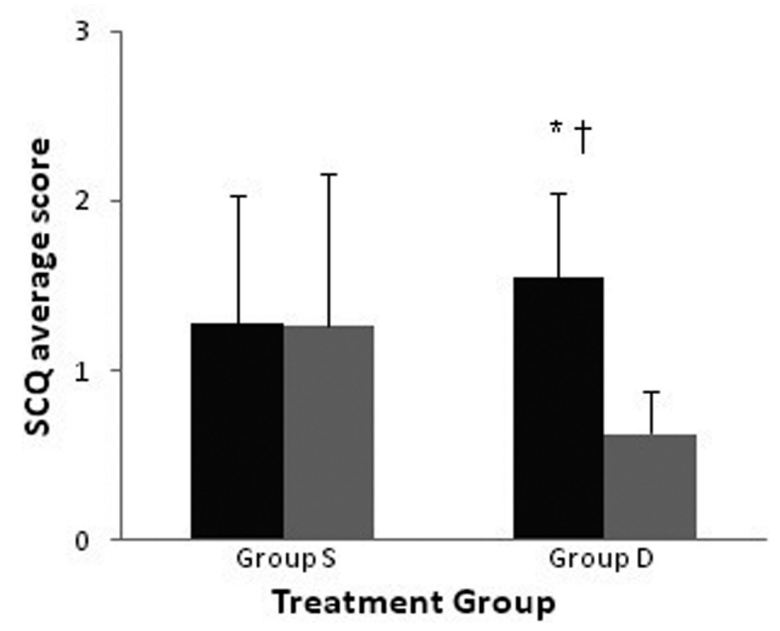

Fig. 2. Mean and SD of Situational Characteristic Questionnaire scores for assessment of visual vertigo symptoms pre (black bars) and post (grey bars) treatment for Groups S and D. * indicates a significant within-group difference $(p<0.05)$; ${ }^{\dagger}$ indicates a significant difference between groups $(p<0.05)$.

\subsection{Total phobia}

No significant between-group differences were noted. However, a significant within-group improvement was noted for Group D1 $(Z=-2.03, p=0.04)$ where all patients' scores improved compared to $3 / 5$ for Group D and 6/11 for Group S.

\subsection{Dynamic gait index}

No significant between- or within-group differences were noted (Tables 3, 4). For both Groups D and D1 3/5 patients improved while 2 patients in each group with a maximum score at baseline showed no change. For Group S, 4/11 patients improved, while others showed no change (including 1 with maximum score) or decreased performance $(n=1)$.

\subsection{Virtual reality cumulative exercise symptom ratings (VRCESS)}

A significant difference was noted between Groups $\mathrm{D}$ and $\mathrm{S}(U=4.0, z=-2.68, p=0.01)$ with a $59 \%$ improvement for the former compared to $7.2 \%$ for the latter. A significant difference was also noted between the participants who only completed Group $\mathrm{S}$ and Group D1 with a $65.2 \%$ improvement for the latter compared to a $6.5 \%$ improvement for the former ( $U=0.00, z=-2.57, p=0.01$ ). Within-group analysis showed a significant difference for Group D
( $Z=-2.04, p=0.04)$; however results for Group D1 only showed a trend towards significance $(Z=-1.83$, $p=0.07)$.

\section{Discussion}

This pilot study assessed the potential benefit of combining vestibular exercises with exposure to a wide field-of-view dynamic, virtual environment on VV symptoms in patients with chronic symptoms secondary to a peripheral vestibular disorder. Results indicated significant improvements for VV symptoms for both Groups D and D1 although the improvements were greater for the former. Both Groups D and D1 showed a greater than $50 \%$ improvement in subjective symptom scores for the exercises practiced during dynamic visual exposure. Only Group S showed a significant improvement for depression scores. Although there was a significant difference for symptom duration between Groups S and D, as in previous studies no relationship was noted between symptom duration and age with self-report measures $[8,17,31,44]$. This discussion will comprise three sections: a) VV; b) psychological state; and c) gait.

\subsection{Visual vertigo}

VV symptoms improved by $59 \%$ and $28 \%$ respectively for Groups D and D1 compared to virtually no change for Group S. Although the difference in VV symptom score change was greater for Group D compared to Group D1, this was not statistically significant and only showed a trend towards significance. This discrepancy is most likely due to the low subject numbers and higher variability in SCQ scores for Group D1 as indicated by the standard deviation. Overall these findings indicate that virtual reality providing a dynamic wide field-of-view is an alternative visual device to optokinetic stimulation, and can be included as a useful beneficial adjunct to treatment.

Improvements were noted both for VV symptoms and symptoms provoked for the exercises practised while viewing the dynamic virtual reality scene. It is hypothesized that the basis for visual motion stimulation, including virtual reality, is habituation, a decrease in response magnitude to repetitive, symptom provoking, sensory stimulation $[10,29,43]$ and neural adaptability [32]. During PET and fMRI studies exposure to visual optokinetic stimulation, in the absence of vestibular stimulation, leads to consistent activation 
of cortical areas related to visual motion processing and eye movement control, and deactivation of parietoinsular vestibular cortices indicating a reciprocally inhibitory visual-vestibular interaction $[6,11,21]$. These findings have been interpreted as indicative of a functionally relevant sensory re-weighing process. In view of the present study's findings, we would similarly propose that dynamic virtual reality exposure promotes a reduced visual reliance and thus fosters a more effective use of vestibulo-proprioceptive cues, possibly through sensory re-weighting [37]. Various authors have suggested that virtual reality may be an ideal method of optokinetic stimulation as the stimulus can closely reflect conditions found in everyday symptom provoking environments (i.e. grocery store aisle) and the response can be customized according to the patients needs [38, 43].

The crowded square virtual environment used in this study (developed by Dr. Franco Tecchia) was one participants could easily relate to and which is often reported as symptom provoking. Although the quality of rendering presented to participants within the IPT was on par with that seen with most computer games, the life-sized scene containing life-sized virtual characters, the rapidly updated and normal behaviour of the environment and moving figures, together with a wide field-of-view, stereo and head-tracking, quickly drew participants into the virtual environment. Although no subjective nor objective measures were available to assess levels of presence [36] the behaviour exhibited by both Groups D and D1 participants suggests they experienced a high degree of presence and acted as they would for a similar real-life scenario (e.g. moving to avoid both members of the computer generated crowd and computer generated walls). It has been suggested that the greater level of presence with a street or grocery aisle scene, in contrast to those employed in some pilot studies (a panoramic view or a checkered pattern), may play a key role in the rehabilitation potential of virtual reality $[39,43]$.

It is clear from this study and others [31,32] that objective visual dependency and VV symptoms only improve when vestibular exercises are combined with exposure to dynamic visual motion. However, improvements in objective visual dependency [32] in healthy controls and VV symptoms experienced in various environments (i.e. supermarket aisles, watching moving traffic, scrolling on the computer) in patients with a peripheral vestibular disorder [3] have also been noted with optokinetic stimulation which does not closely reflect everyday environments. Future studies are required to compare the different types of optokinetic stimulation available. Furthermore, in regards to virtual reality, wide field-of-view devices are expensive, space consuming, and non-portable. Therefore the transfer of this technique into everyday clinical practice may be limited and difficult. Head mounted virtual reality devices offering a narrow field-of-view have been suggested as the most likely choice for vestibular rehabilitation as they overcome the limitations above [38].

\subsection{Psychological state}

Two-thirds of patients with vestibular symptoms referred to a tertiary referral centre also suffer from psychological symptoms including anxiety, depression and phobic disorders including agoraphobia [18,27]. Whilst the Beck Anxiety and Depression scores for participants included in this study did not indicate a substantial psychological overlay, as would be expected from the exclusion criteria, a significant decline in depression scores was noted for Group S only while a trend towards significance was noted for anxiety scores for Group D, which was most likely affected by the low participant numbers for this group. Baseline and post-intervention total phobia scores were also within normal ranges for all groups [28], however a significant within-group improvement was noted for Group D1. It is likely that these results were also affected by participant numbers and within-group variability as the pre-post score change was actually greater for Group D1. These findings are not surprising as the benefit of controlled exposure to static and dynamic virtual reality systems has been demonstrated in the treatment of post traumatic stress disorder [12] fear of flying [24, $34]$ or public speaking, social phobia [22], fear [3,19] and agoraphobia [40].

\subsection{Dynamic gait index}

Outcome measures able to detect clinically significant changes over time are necessary in determining an intervention's efficacy [14]. The DGI has been used as a measure of functional gait and falls risk in a number of studies $[15,41,42]$ and a 3 -point change in DGI score has been identified as clinically significant in patients with peripheral vestibular dysfunction [15]. Although no significant between or within-group differences were noted for the DGI, Group D did achieve a 3 point change while the other groups did not. This however may be due to the fact that study participants were young and scored highly at baseline with average group scores within normal ranges for falls risk. 


\section{Conclusions}

Despite the limited subject numbers, the results of this pilot study indicate that VV symptoms significantly improve from a rehabilitation program combining vestibular exercises with exposure to a challenging dynamic virtual reality environment. Further studies are required with greater participant numbers to investigate the true efficacy of virtual reality devices in vestibular rehabilitation with regards to treatment outcome, long term efficacy and cost.

\section{Acknowledgments}

We would like to thank Dr. R. Davies for her help in recruiting participants and Dr. Peter Milligan for statistical advice. Financial assistance provided by the Ear, Nose and Throat Department of the Royal Sussex County Hospital, Brighton is gratefully acknowledged.

\section{References}

[1] M.W.A. Shumway-Cook, Motor Control: Theory and Practical Applications, Williams and Wilkins, Baltimore, MD, 1995.

[2] R.A.S.A.T. Beck, Manual for the Beck Depression Inventory, Psychological Corporation, San Antonio, TX, 1993.

[3] Y. Agrawal, J.P. Carey, C.C. Della Santina, M.C. Schubert and L.B. Minor, Disorders of balance and vestibular function in US adults: Data from the National Health and Nutrition Examination Survey, 2001-2004, Arch Intern Med 169 (2009), 938-944.

[4] A. Bisdorff, M. Von Brevern, T. Lempert and D.E. NewmanToker, Classification of vestibular symptoms: Towards an international classification of vestibular disorders, $J$ Vestib Res 19 (2009), 1-13.

[5] F.O. Black, C.R. Angel, S.C. Pesznecker and C. Gianna, Outcome analysis of individualized vestibular rehabilitation protocols, Am J Otol 21 (2000), 543-551.

[6] T. Brandt, P. Bartenstein, A. Janek and M. Dieterich, Reciprocal inhibitory visual-vestibular interaction. Visual motion stimulation deactivates the parieto-insular vestibular cortex, Brain 121(Pt 9) (1998), 1749-1758.

[7] A.M. Bronstein, Visual vertigo syndrome: Clinical and posturography findings, J Neurol Neurosurg Psychiatry 59 (1995), 472-476.

[8] K.E. Brown, S.L. Whitney, D.M. Wrisley and J.M. Furman, Physical therapy outcomes for persons with bilateral vestibular loss, Laryngoscope 111 (2001), 1812-1817.

[9] D.S.C. Cruz-Neira and T. DeFanti, Surround-screen projection-based virtual reality: Design and implementation of the CAVE, in: Proceedings of SIGGRAPH, 1993, pp. 135-142.

[10] T.E. Cawthorne, The physiological basis for head exercises, $J$ Chart Soc Physiother 30 (1944), 106-107.

[11] M. Dieterich, S. Bense, T. Stephan, T.A. Yousry and T. Brandt, fMRI signal increases and decreases in cortical areas during small-field optokinetic stimulation and central fixation, Exp Brain Res 148 (2003), 117-127.
[12] J. Difede, J. Cukor, N. Jayasinghe, I. Patt, S. Jedel, L. Spielman, C. Giosan and H.G. Hoffman, Virtual reality exposure therapy for the treatment of posttraumatic stress disorder following September 11, 2001, J Clin Psychiatry 68 (2007), 1639-1647.

[13] M. Guerraz, L. Yardley, P. Bertholon, L. Pollak, P. Rudge, M.A. Gresty and A.M. Bronstein, Visual vertigo: Symptom assessment, spatial orientation and postural control, Brain 124 (2001), 1646-1656.

[14] G. Guyatt, S. Walter and G. Norman, Measuring Change over Time - Assessing the Usefulness of Evaluative Instruments, Journal of Chronic Diseases 40 (1987), 171-178.

[15] T.D. Hall, M.C. Schubert and S.J. Herdman, Prediction of fall risk reduction as measured by dynamic gait index in individuals with unilateral vestibular hypofunction, Otology and Neurotology 25 (2004), 746-751.

[16] S.J. Herdman, M.C. Schubert, V.E. Das and R.J. Tusa, Recovery of dynamic visual acuity in unilateral vestibular hypofunction, Arch Otolaryngol Head Neck Surg 129 (2003), 819-824.

[17] F.B. Horak, C. Jones-Rycewicz, F.O. Black and A. ShumwayCook, Effects of vestibular rehabilitation on dizziness and imbalance, Otolaryngol Head Neck Surg 106 (1992), 175-180.

[18] R.G. Jacob, Panic disorder and the vestibular system, Psychiatr Clin North Am 11 (1988), 361-374.

[19] L.K. James, C.Y. Lin, A. Steed, D. Swapp and M. Slater, Social anxiety in virtual environments: Results of a pilot study, Cyberpsychology and Behavior 6 (2003), 237-243.

[20] E.A. Keshner, J. Streepey, Y. Dhaher and T. Hain, Pairing virtual reality with dynamic posturography serves to differentiate between patients experiencing visual vertigo, J Neuroeng Rehabil 4 (2007), 24.

[21] A. Kleinschmidt, K.V. Thilo, C. Buchel, M.A. Gresty, A.M. Bronstein and R.S. Frackowiak, Neural correlates of visualmotion perception as object-or self-motion, Neuroimage 16 (2002), 873-882.

[22] E. Klinger, S. Bouchard, P. Legeron, S. Roy, F. Lauer, I. Chemin and P. Nugues, Virtual reality therapy versus cognitive behavior therapy for social phobia: A preliminary controlled study, Cyberpsychol Behav 8 (2005), 76-88.

[23] O.T. Leyfer, J.L. Ruberg and J. Woodruff-Borden, Examination of the utility of the Beck Anxiety Inventory and its factors as a screener for anxiety disorders, J Anxiety Disord 20 (2006), 444-458.

[24] N. Maltby, I. Kirsch, M. Mayers and G.J. Allen, Virtual reality exposure therapy for the treatment of fear of flying: A controlled investigation, J Consult Clin Psychol 70 (2002), 1112-1118.

[25] I.M. Marks and A.M. Mathews, Brief standard self-rating for phobic patients, Behav Res Ther 17 (1979), 263-267.

[26] C.A. McGibbon, D.E. Krebs, S.W. Parker, D.M. Scarborough, P.M. Wayne and S.L. Wolf, Tai Chi and vestibular rehabilitation improve vestibulopathic gait via different neuromuscular mechanisms: Preliminary report, BMC Neurol 5 (2005), 3.

[27] L. McKenna, R.S. Hallam and R. Hinchcliffe, The prevalence of psychological disturbance in neurotology outpatients, Clin Otolaryngol Allied Sci 16 (1991), 452-456.

[28] J.S. Mizes and J. Crawford, Normative Values on the Marks and Mathews Fear Questionnaire - a Comparison as a Function of Age and Sex, Journal of Psychopathology and Behavioral Assessment 8 (1986), 253-262.

[29] M.E. Norre and W. De Weerdt, Treatment of vertigo based on habituation. 2. Technique and results of habituation training, J Laryngol Otol 94 (1980), 971-977. 
[30] M. Pavlou, R.A. Davies and A.M. Bronstein, The assessment of increased sensitivity to visual stimuli in patients with chronic dizziness, J Vestib Res 16 (2006), 223-231.

[31] M. Pavlou, A. Lingeswaran, R.A. Davies, M.A. Gresty and A.M. Bronstein, Simulator based rehabilitation in refractory dizziness, J Neurol 251 (2004), 983-995.

[32] M. Pavlou, C. Quinn, K. Murray, C. Spyridakou, M. Faldon and A.M. Bronstein, The effect of repeated visual motion stimuli on visual dependence and postural control in normal subjects, Gait Posture 33 (2011), 113-118.

[33] S.O.L. R.G. Jacob, J.M.R. Furman, J.D. Durrant and S.M. Turner, Panic disorder with vestibular dysfunction: Further clinical observations and description of space and motion phobi stimuli, J Anxiety Disord 3 (1989), 117-130.

[34] B.O. Rothbaum, L. Hodges, S. Smith, J.H. Lee and L. Price, A controlled study of virtual reality exposure therapy for the fear of flying, J Consult Clin Psychol 68 (2000), 1020-1026.

[35] P.J.S. S.L. Whitney, K. Brown, J.M. Furman, J.L Jacobson and M.S. Redfern, The Potential Use of Virtual Reality in Vestibular Rehabilitation: Preliminary Findings with the BNAVE Neurology Report 26 (2002), 72-78.

[36] M.V. Sanchez-Vives and M. Slater, From presence to consciousness through virtual reality, Nature Reviews Neuroscience 6 (2005), 332-339.

[37] A. Shumway-Cook and F.B. Horak, Rehabilitation strategies for patients with vestibular deficits, Neurol Clin 8 (1990), 441-457.
38] P.J. Sparto, J.M. Furman, S.L. Whitney, L.F. Hodges and M.S. Redfern, Vestibular rehabilitation using a wide field of view virtual environment, Conf Proc IEEE Eng Med Biol Soc 7 (2004), 4836-4839.

[39] E. Viirre and R. Sitarz, Vestibular rehabilitation using visual displays: Preliminary study, Laryngoscope 112 (2002), 500503.

[40] F. Vincelli, H. Choi, E. Molinari, B.K. Wiederhold, S. Bouchard and G. Riva, Virtual reality assisted cognitive behavioral therapy for the treatment of panic disorders with agoraphobia, Medicine Meets Virtual Reality 02/1085 (2002), 552559.

[41] S. Whitney, D. Wrisley and J. Furman, Concurrent validity of the Berg Balance Scale and the Dynamic Gait Index in people with vestibular dysfunction, Physiother Res Int 8 (2003), 178186.

42] S.L. Whitney, M.T. Hudak and G.F. Marchetti, The dynamic gait index relates to self-reported fall history in individuals with vestibular dysfunction, J Vestib Res 10 (2000), 99-105.

[43] S.L. Whitney, P.J. Sparto, L.F. Hodges, S.V. Babu, J.M. Furman and M.S. Redfern, Responses to a virtual reality grocery store in persons with and without vestibular dysfunction, Cyberpsychol Behav 9 (2006), 152-156.

[44] S.L. Whitney, D.M. Wrisley, G.F. Marchetti and J.M. Furman, The effect of age on vestibular rehabilitation outcomes, Laryngoscope 112 (2002), 1785-1790. 